\title{
Feto-maternal immune regulation by TIM-3/galectin-9 pathway and PD-1 molecule in mice at day 14.5 of pregnancy
}

\author{
Matyas Meggyes a, b, Adrienn Lajko a , Tamas Palkovics ${ }^{\mathrm{a}, \mathrm{b}}{ }^{\mathrm{b}}$, Anett Totsimon ${ }^{\mathrm{a}}$, Zsolt Illes ${ }^{\mathrm{c}, \mathrm{d}}$,

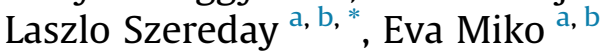 \\ a University of Pecs, Clinical Centre, Department of Medical Microbiology and Immunology, Pecs, Hungary \\ b Janos Szentagothai Research Centre, Pecs, Hungary \\ ${ }^{c}$ Department of Neurology, Odense University Hospital, Odense, Denmark \\ ${ }^{\mathrm{d}}$ Institute of Clinical Research, University of Southern Denmark, Odense, Denmark
}

\section{A R T I C L E I N F O}

\section{Article history:}

Received 19 May 2015

Received in revised form

8 July 2015

Accepted 13 July 2015

\section{Keywords:}

TIM-3

Gal-9

PD-1

Pregnancy

Feto-maternal interface

Immunity

\begin{abstract}
A B S T R A C T
Introduction: Immunoregulation implies the activation of negative pathways leading to the modulation of specific immune responses. Co-inhibitory receptors (such as PD-1 and TIM-3) represent possible tools for this purpose. PD-1 and TIM-3 have been demonstrated to be present on immune cells suggesting general involvement in immunosuppression such as fetomaternal tolerance. The aim of our study was to investigate the expression pattern of PD-1, TIM-3, and its ligand Gal- 9 on different immune cell subsets in the peripheral blood and at the fetomaternal interface in pregnant mice.

Methods: TIM-3 and PD-1 expression by peripheral and decidual immune cells from pregnant BALB-c mice in 2 weeks of gestational age were measures by flow cytometry. Placental galectin- 9 expression was determined by immunohistochemically and RT-PCR.

Results: Gal-9 was found to be present in the spongiotrophoblast layer of the haemochorial placenta. Decidual NK, NKT and $\gamma / \delta$ T cells showed increased PD-1 expression and reduced cytotoxic potential when compared to the periphery. TIM-3 expression by NK cells and $\gamma / \delta$ T cells is similar both in the periphery and in the decidua, notably, their relative TIM-3 expression is increased locally which is associated with reduced lytic activity. Decidual NKT cells exhibit a reduced TIM-3 expression with increased relative receptor expression and a slightly increased cytotoxicity when compared to the periphery.

Discussion: Our data reveals a particularly complex, tissue and cell type specific immunoregulatory mechanism by the investigated co-inhibitory receptors at the fetomaternal interface.
\end{abstract}

๑) 2015 Elsevier Ltd. All rights reserved.

\section{Introduction}

During pregnancy, immunological recognition of the semiallogenic fetus by the maternal immune system leads to a unique immune response phenomenon. At the very early stage of pregnancy, immunotolerance of the fetus has to be synchronized with decidual inflammatory processes required for successful

Abbreviations: DC, dendritic cell; Gal-9, galectin-9; Gd, gestation day; PD-1, programmed cell death-1; RPMI 1640, Roswell Park Memorial Institute formulation 1640; QRT-PCR, quantitative real-time PCR; TIM-3, T cell immunoglobulion and mucin domain 3.

* Corresponding author. University of Pecs, Clinical Centre, Department of Medical Microbiology and Immunology, Pecs, Hungary.

E-mail address: szereday.laszlo@pte.hu (L. Szereday). implantation and placentation of the embryo. Later on, besides allowing fetal development the maternal immune system should be able to generate adequate responses to infections or eradicate nascent tumor cells. Therefore, controlling and regulating contradictory immune effector functions during pregnancy portrays a key role in maintaining immune homeostasis. Immunoregulation is mediated by a complex network of cellular and molecular interactions with the predominant role of regulatory T cells as cellular components of the immune system and an increased number of molecules with immunoregulatory capacity.

TIM-3 was first identified as a receptor specifically expressed by terminally differentiated IFN- $\gamma$ producing CD4 + T helper 1 (Th1) and on CD8+ T cytotoxic cells [1]. Several studies demonstrated the expression of TIM-3 on a variety of immune cells including Th1, Th17, NK cells, NKT cells, CD4+CD25+ T cells (Tregs) and on cells of 
the innate immune system, including dendritic cells (DCs), macrophages [2], and mast cells [3]. Mounting evidence supports the crucial role of TIM-3 in regulating immune responses [4] but the in vivo function of this receptor is still poorly understood. Using an allogeneic mouse model of pregnancy Chabtini et al. examined the TIM-3 expression on antigen presenting myeloid cells and indicated their possible role in the regulation of tolerance at the fetomaternal interface [5].

Galectin-9 (Gal-9), an endogenous lectin, was originally described as a potent eosinophil chemoattractant. Gal-9 can influence the immune system in different ways [6], either by exacerbating the inflammatory process [7] or by exhibiting therapeutic effects in autoimmune disease models, such as autoimmune arthritis, experimental allergic encephalomyelitis, and type 1 diabetes mellitus. Notably, it is generally believed that the engagement of Gal-9 by its receptor TIM-3 established the Gal-9/TIM-3 pathway as a novel regulator of Th1 immunity and tolerance induction [8].

TIM-3 is the most extensively studied Gal-9 receptor. A growing body of evidence proves that the engagement of these two molecules leads to the death of Th1 and Th17 cells thus negatively regulates IFN $-\gamma$ secretion, furthermore influences the ability to induce $\mathrm{T}$ cell tolerance and triggers a significant signal cascade to induce apoptosis of Th1 type immune cells in both mice and humans [9-11]. Therefore, the connection between TIM-3 and Gal9 may function as a negative regulator, dampening Th1- and Th17 driven immune responses and inducing peripheral tolerance by modulating the Th1/Th2 balance [12].

The TIM-3 mediated immunomodulation was first described as a clearly negative regulatory mechanism inducing immunotolerance and T cell apoptosis [13]. However, there are conflicting data surrounding the TIM-3 mediated regulation of innate immunity. In addition to suggesting similar immunosuppressive actions of TIM-3 on innate immune cells, as observed in T cells [14,15], TIM-3 was also found to promote innate immune responses $[2,16]$. Moreover, several recently published papers report that in reference to viral infections, overexpression of TIM-3 on NK cells was associated with effector dysfunction $[17,18]$.

Programmed cell death-1 (PD-1) is a receptor and a member of the B7/CD28 family. It is known to downregulate $\mathrm{T}$ cell functions. PD-1 expression was observed on CD4+ Th- and CD8+ T lymphocytes, B lymphocytes, Tregs, NK, NKT cells, DCs, and activated monocytes [19]. PD-1 is not expressed on resting T cells but is inducible upon activation [20]. Known ligands of PD-1 include PDL1 [21,22] and PD-L2 [23]. The binding of PD-1 on T cells with its ligand PD-L1 leads to decreased cytokine production and negatively regulates $\mathrm{T}$ cell proliferation and cell lysis during immune responses to pathogens or cancer [24]. Many publications revealed and characterized PD-1 as a classical exhaustion marker of T cells with poor effector functions $[25,26]$.

Since pregnancy represents a unique model of local immunotolerance, regulatory pathways exerted by these co-inhibitory molecules could have significant impact on maternal immunosuppression. Therefore, the aim of our study was to investigate the expression pattern of TIM-3, PD-1 and Gal-9 on different immune cell subsets in the peripheral blood and at the fetomaternal interface.

\section{Materials and methods}

\subsection{Animal model}

Young (2 months) BALB-c mice were purchased from the Pécs Experimental Central Animal Laboratory and maintained on a $12 \mathrm{~h}$ light/dark cycle at $20-22{ }^{\circ} \mathrm{C}, 40-60 \%$ humidity, and were fed with standard feed pellets and tap water. Potential mates were paired up each evening and the presence of the copulatory plug was examined next morning. Once a plug is detected it is considered the gestation day (gd) 0.5 . Gravid females were killed on gd 14.5 by cervical dislocation, the spleen and the uterine horns were aseptically removed. Animal housing, care, and application of experimental procedures were in accordance with institutional guidelines under approved protocols (No. BA02/2000-20/2006, University of Pecs).

\subsection{Isolation of mononuclear cells from the spleen}

Spleens were homogenized thoroughly with a syringe plunger, and single-cell suspensions were prepared using a 70- $\mu \mathrm{m}$ nylon cell strainer (BD Pharmingen). Subsequently, cells were washed in phosphate-buffered saline (PBS). Supernatant was aspirated and the pellet was resuspended in PBS and filtered via 40- $\mu$ m nylon cell strainer (BD Pharmingen). Mononuclear cells were isolated on Ficoll-Paque (GE Healthcare) gradient. Cells were collected and resuspended in RPMI 1640 (Lonza) supplemented with penicillin (Lonza), streptomycin (Lonza) and 10\% heat inactivated fetal calf serum (FCS) (Gibco).

\subsection{Isolation of mononuclear cells from the decidua}

The conceptus with the placentae was removed from the surrounding area of the endometrial tissue. Then the decidua was separated from the isolated placentae, sliced with scissors and finally digested with collagenase (Sigma-Aldrich) for $30 \mathrm{~min}$ at $37{ }^{\circ} \mathrm{C}$. Placentas were removed and divided into pieces for RNA isolation followed by quantitative real-time PCR (qRT-PCR) and complete implantations were frozen for immunohistochemistry. The decidua next were homogenized thoroughly with a syringe plunger, and single-cell suspensions were prepared using a $70-\mu \mathrm{m}$ nylon cell strainer. Subsequently, cells were washed in RPMI 1640 supplemented with penicillin, streptomycin and 10\% FCS. The supernatant was aspirated and the pellet was resuspended in PBS and filtered via $40-\mu \mathrm{m}$ nylon cell strainer. Mononuclear cells were isolated on Ficoll-Paque gradient. Cells were collected and resuspended in RPMI 1640.

\subsection{Labeling of mononuclear cell and flow cytometric analysis}

The isolated mononuclear cells $\left(1 \times 10^{6}\right.$ in $100 \mu \mathrm{l}$ PBS/tube) were incubated for $30 \mathrm{~min}$ at room temperature (RT) with fluorochromelabeled monoclonal antibodies. Following surface staining, the cells were washed with PBS, resuspended in $300 \mu \mathrm{l}$ PBS containing 1\% paraformaldehyde (PFA) and stored at $4{ }^{\circ} \mathrm{C}$ in the dark until fluorescence-activated cell sorting (FACS) analysis. Labeled cells were analyzed with a FACSCalibur flow cytometer (BD Immunocytometry Systems, Erembodegen, Belgium) equipped with the CellQuest software program (BD Biosciences, San Diego, CA, USA) and a BD FACSCanto II flow cytometer (BD Immunocytometry Systems, Erembodegen, Belgium) with the BD FACSDiva V6. software for data acquisition and analysis.

\subsection{Antibodies}

The following monoclonal antibodies were implemented:

Brilliant Violet (BV) 421-conjugated anti-mouse PD-1 (BD Pharmingen), BV510-conjugated anti-mouse CD3 (BD Pharmingen), BV510-conjugated anti-mouse $\gamma / \delta$ (BD Pharmingen), fluorescein isothiocyanate (FITC)-conjugated anti-mouse CD4 (BD Pharmingen), FITC-conjugated anti-mouse CD8 (BD Pharmingen), FITCconjugated anti-mouse CD107a (BD Pharmingen), FITCconjugated anti-mouse CD49b (BD Pharmingen), FITC-conjugated 
anti-mouse $\gamma / \delta$ (BD Pharmingen), phycoerythrin (PE)-conjugated anti-mouse $\gamma / \delta$ (BD-Pharmingen), PE-conjugated anti-mouse TIM3 (R and D Systems), PE-conjugated anti-mouse Gal-9 (Biolegend), PE-conjugated anti-mouse CD49b (BD Pharmingen), PE-conjugated anti-mouse CD8 (BD Pharmingen), peridinin chlorophyll a protein (PerCP)-conjugated anti-mouse CD3 (BD Pharmingen), PerCPconjugated anti-mouse CD45 (BD Pharmingen), PE-Cy7conjugated anti-mouse CD25 (BD Pharmingen), allophycocyanin (APC)-conjugated anti-human TIM-3 ( $\mathrm{R}$ and D Systems), APCconjugated anti-human FoxP3 (eBioscience) and APC-H7conjugated anti-human CD8 (BD Pharmingen). Control antibodies included isotype-matched FITC-conjugated, PE-conjugated, PerCPconjugated and APC-conjugated rat antibodies.

\subsection{FoxP3 staining}

Following surface labeling intracellular staining of FoxP3 was performed using the FoxP3 Staining Buffer Set (eBioscience) accordance with the manufacture's protocol. Briefly, cells were permeabilized in $1 \mathrm{ml}$ fixation/permeabilization buffer (Concentrate/Diluent 1:4) for $1 \mathrm{~h}$ at $4{ }^{\circ} \mathrm{C}$. Then the samples were washed twice in buffer and stained with the APC-conjugated anti-mouse FoxP3 monoclonal antibody for $1 \mathrm{~h}$ at $4{ }^{\circ} \mathrm{C}$. Flow cytometric analysis was performed on FACSCalibur flow cytometer equipped with the CellQuest software and a BD FACSCanto II flow cytometer with the BD FACSDiva V6. software for data acquisition and analysis.

\subsection{CD107a functional assay}

To determine CD107a surface expression, the cells were incubated for $4 \mathrm{~h}$ at $37{ }^{\circ} \mathrm{C}$ in the presence of FITC-conjugated antimouse CD107a monoclonal antibody in RPMI 1640 medium containing 10\% FCS, penicillin, streptomycin, ionomycin (Sigma-Aldrich) and phorbol myristate acetate (PMA), (Sigma-Aldrich). The cells were washed and resuspended in PBS then incubated with PE-conjugated anti-mouse $\gamma / \delta$, PE-conjugated anti-mouse CD8, PEconjugated anti-mouse CD49b and PerCP-conjugated anti-mouse CD3 $\mathrm{mAb}$ for $30 \mathrm{~min}$ at room temperature in complete darkness. The cells were washed in PBS, fixed with 1\% PFA and evaluated by FACS

\subsection{PMA/ion treatment}

The isolated peripheral and decidual mononuclear cells were treated in 96-well plates. The cells were diluted to a final concentration of $2 \times 10^{5}$ cells $/ 0.2 \mathrm{ml}$ in RPMI 1640 supplemented with Lglutamine, penicillin and streptomycin and washed for $7 \mathrm{~min}$ and centrifuged at 1200 RPM. The supernatant was aspirated and the cells were resuspended in RPMI 1640 supplemented with $10 \%$ FCS, P, S, PMA ( $1 \mathrm{ng} / \mathrm{ml}$ ), Ion (50 ng/ml) and incubated for $24 \mathrm{~h}$ at $37^{\circ} \mathrm{C}$. After incubation the plates were centrifuged at $1200 \mathrm{RPM}$ for $7 \mathrm{~min}$. The cell free supernatants were collected, aliquoted and stored at $-80{ }^{\circ} \mathrm{C}$ in mechanical freezer for additional cytokine analyses.

\subsection{Immunohistochemistry (IHC)}

Placental tissue sections were embedded in paraffin and $4 \mu \mathrm{m}$ thick sections were prepared after being fixed with $4 \%$ neutral formalin for more than $24 \mathrm{~h}$. After being deparaffinized in xylol, three times in each for $5 \mathrm{~min}$ and rehydrated in degraded alcohol series $(96 \%, 80 \%, 70 \%, 50 \%)$ for $5 \mathrm{~min}$ each, the mouse paraffinembedded tissue sections were washed in distilled water $\left(\mathrm{dH}_{2} \mathrm{O}\right)$. Afterward the antigen retrieval was performed in boiling citrate buffer for 20 min (1:10; pH 6.0-6.2; Dako). Next, the samples were cooled down to RT and a double washing step was performed with
$\mathrm{dH}_{2} \mathrm{O}$. During the blocking of endogenous peroxidase the samples were kept for $15 \mathrm{~min}$ in $3 \% \mathrm{H}_{2} \mathrm{O}_{2}$, which was diluted in TBS. The revealing was finished by a double washing step in $\mathrm{dH}_{2} \mathrm{O}$. The samples were washed twice with TBS supplemented with Tween (0.05\%, pH 7.4) for $5 \mathrm{~min}$ and pre-blocked with 3\% BSA for $20 \mathrm{~min}$. The tissue sections were washed with TBST for 5-10 min and incubated with biotinylated anti mouse Galectin- 9 primary antibody $(1: 10 ; \mathrm{R} \& \mathrm{D})$ for $1 \mathrm{~h}$ at RT. After washing the slides three times in TBST the samples were incubated with Streptavidin-Biotinylated Horseradish Peroxidase Complex (1:100; GE Healthcare) for $30 \mathrm{~min}$ at RT. Following the washing procedure (three times), the signal was detected with Liquid DAB + Substrate Chromogen System (Dako) for 5-30 min at RT. In an effort to stop and pause the reaction, the slides were positioned in ultrapure water followed by two distinct rinse cycles in $\mathrm{dH}_{2} \mathrm{O}$ was performed. Hematoxylin counterstain was performed for $3 \mathrm{~min}$ at RT then rinsed three times with tap water. During the final step, the slides were covered with gelatin-glycerol solution.

Histological evaluation was performed by a pathologist generating a qualitative assessment on all tissues sections.

\subsection{RNA isolation, cDNS synthesis, $q R T-P C R$}

Placental tissues and resorptions from mice were homogenized with tissue homogenizer (Sigma-Aldrich). Total RNA was isolated using RNeasyProtect Mini kit (Qiagen) accordance with the manufacturer's protocol. RNA concentration and purity was checked by spectrophotometry using a Nanodrop ND-1000 (Thermo Fisher Scientific). Extracted RNA was reverse transcribed into complementary DNA (cDNA) using the High Capacity RNA-to-cDNA kit (Applied Biosystems). The differential expression of Gal-9 gene was confirmed by using qRT-PCR method. The qRT-PCR examinations were implemented using an SYBR Select Master Mix (Applied Biosystems) and a Rotor-Gene RG-3000 thermal cycler system (Corbett Research). QuantiTect primer assays were purchased from Qiagen for the genes LGALS9 as target and GAPDH as a housekeeping gene. Specificity of the PCR reaction was controlled by melting point analysis and gelelectrophoresis of the amplified DNA.

The relative changes ( $n$-fold) in the transcription of the examined genes between samples were calculated by Rotor-Gene based on the $2-\Delta \Delta C T$ method. Statistical analysis was performed with the Relative Expression Software Tool (REST) 2009 [27].

\subsection{Statistical analysis}

Statistical analysis was performed using the reliable and preferred statistical software SPSS version 20. Package and the statistical comparisons were made by using the Student's t-tests. Distinctly, notable differences were determined significant if the $\mathrm{p}$ value was equal to or less than 0.05 .

\section{Results}

\subsection{Immunohistochemistry of pregnant mouse placentae.}

We investigated the Gal-9 protein expression at the murine fetomaternal interface on gd 14.5 using immunohistochemistry. As a result, our stainings highlight that we can now effectly detect the Gal-9 molecule in the placentae spongiotrophoblast layer (Fig. 1).

3.2. Gal-9 expression by Treg cells and the proportion of Gal-9+ Th cell population in spleen and decidua.

We analyzed the surface Gal-9 expression by splenic and decidual regulatory $\mathrm{T}$ cells, in pregnant mice. We discovered a 


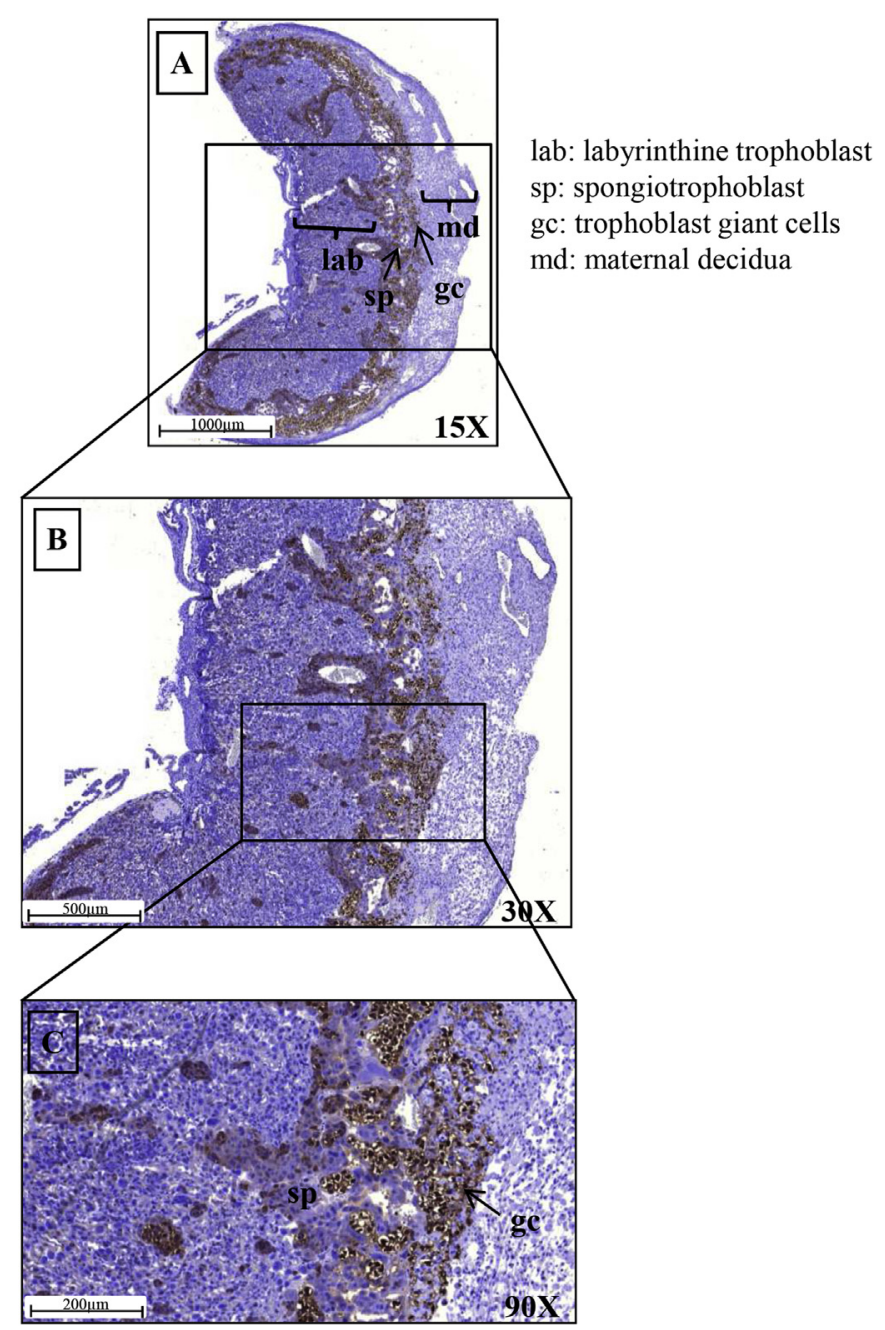

Fig. 1. Immunohistochemical staining of pregnant mouse placentae. IHC was performed on pregnant mouse placentae with anti-Gal-9 antibody and demonstrating the presence and localization of Gal-9 within the spongiotrophoblast layer and in giant cells. Obtaining quantitative data from histological sections represent a more intense staining by trophoblast giant cells compared to spongiotrophoblast layer. The images were captures utilizing the Pannoramic DESK scanner (3DHISTECH Ltd., Hungary). In acquiring the analysis, the Pannoramic viewer software was implemented (3DHISTECH Ltd., Hungary). significantly increase in Gal-9 expression by the decidual Treg cells when compared to the splenic Treg cells (Fig. 2/A).

We could not determine statistical differences in the frequency of Gal-9 positive Th cells in decidua and spleen (Fig. 2/B).

\subsection{Phenotype analysis of splenic and decidual mononuclear cells from pregnant mice in 2 weeks of gestational age.}

In our study, we investigated the percentage of CD4+, CD8+ T cells subpopulations, $\gamma / \delta$ T cells, Treg cells, NK and NKT cells in the spleen and in the decidua of pregnant mice. We observed a significant increase in decidual $\gamma / \delta \mathrm{T}$, NK and NKT cells frequency compared to the periphery while the decidual CD4+, CD8+ T and Treg cells frequency significantly decreased when compared to the periphery (Fig. 3).

\subsection{Differential TIM-3 and PD-1 expression by splenic and decidual mononuclear cell subsets.}

We measured the surface expression of TIM-3 on different lymphocyte subsets by flow cytometry. We observed a significantly decreased TIM-3 expression by decidual NKT cells compared to the periphery. TIM-3 expression showed no statistical difference between the spleen and the decidua by NK and $\gamma / \delta$ T cells (Fig. 4/A).

Furthermore, we also noted a significant increase in receptor density within the decidual NK, NKT and $\gamma / \delta$ T cells when compared with the periphery (Fig. 4/B).

During our investigation of the PD-1 expression by NK cells, $\gamma /$ $\delta \mathrm{T}$ and NKT cells in pregnant mice we discovered that all analyzed cell populations demonstrated an increase in PD-1 expression within the decidua compared to the periphery (Fig. 4/C).

As a result of our investigation of the peripheral and decidual TIM-3/PD1 double positive cells we found a significant decrease in decidual NKT and $\gamma / \delta$ T cells proportion compared with the periphery. Lastly, we could not detect TIM-3/PD-1 double positive NK cells in the examined tissues (Fig. 4/D).

\subsection{Cytotoxic activity of different immune cell subsets in the spleen and in the decidua of pregnant mice.}

The cytotoxic activity was evaluated by measuring the CD107a expression by the investigated cell subsets. We analyzed the CD107a expression by TIM-3+ cell subsets and our results demonstrated a significantly decreased CD107a expression by the decidual TIM-3+ NK and TIM-3+ $\gamma / \delta$ T cells compared to the
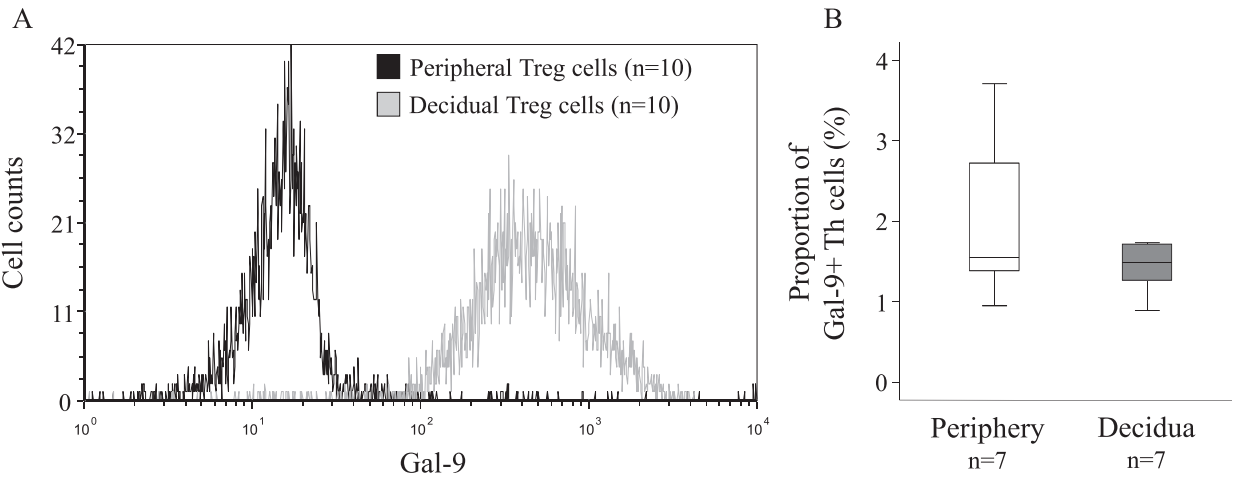

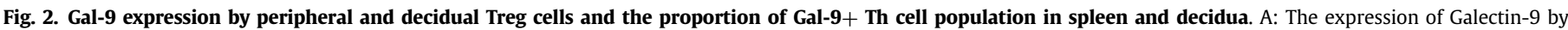

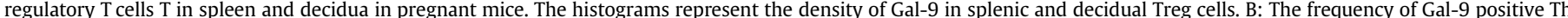

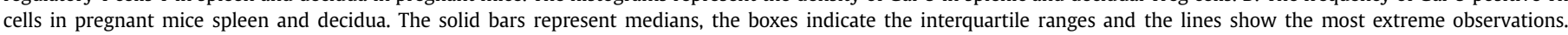
Differences were considered statistically significant for P-values $\leq 0.05$. 


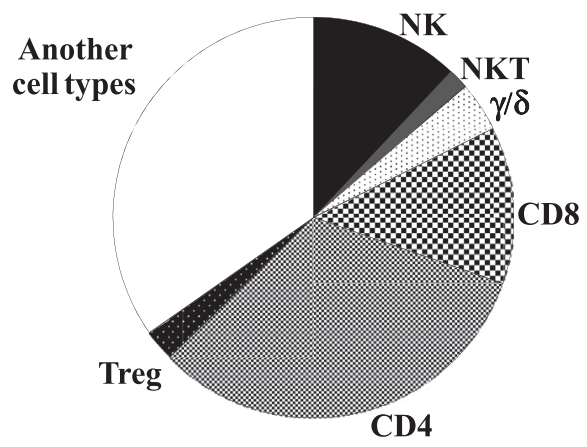

Periphery

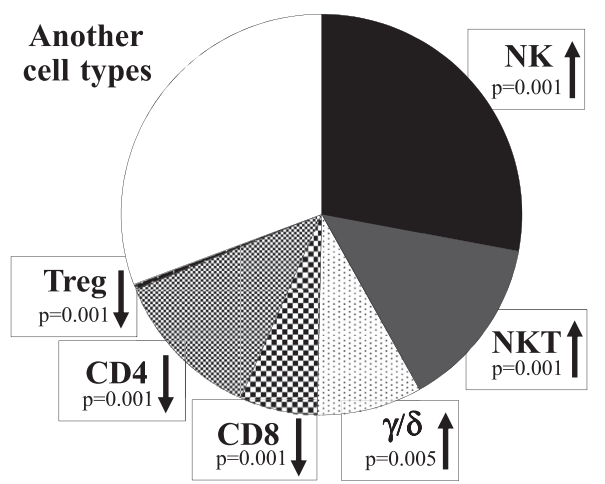

Decidua

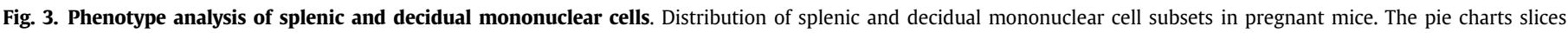
represent each subpopulations rate of 8 determinations, respectively. Differences were considered statistically significant for P-values $\leq 0.05$.

periphery (Fig 5/A). We observed an increasing tendency in the CD107a expression by decidual TIM-3 positive NKT cell which did not attain the level of statistical significance (Fig. 5/A).

Moreover, we analyzed the CD107a expression by the PD-1+ cell populations. Decidual PD-1 positive NK and NKT cells showed significantly decreased cytotoxicity compared to the periphery (Fig. 5/B). Additionally, we discovered a decreased tendency in the cytotoxic activity by decidual PD-1 positive $\gamma / \delta$ T cell which did not reach the level of statistical significance (Fig. 5/B).
3.6. Expression analyses of mice placentas and resorbed embryos Gal-9 mRNA with Quantitative Real Time PCR

To determine the relative expression of LGALS9 in placental tissue and resorbed embryos qRT-PCR analysis was performed with normalization to GAPDH as a housekeeping gene. We tested four resorbed embryos in three different mice, and all resorbed embryos showed a LGALS9 relative expression level of 0.04 compared to normal placental tissue of the same mouse.
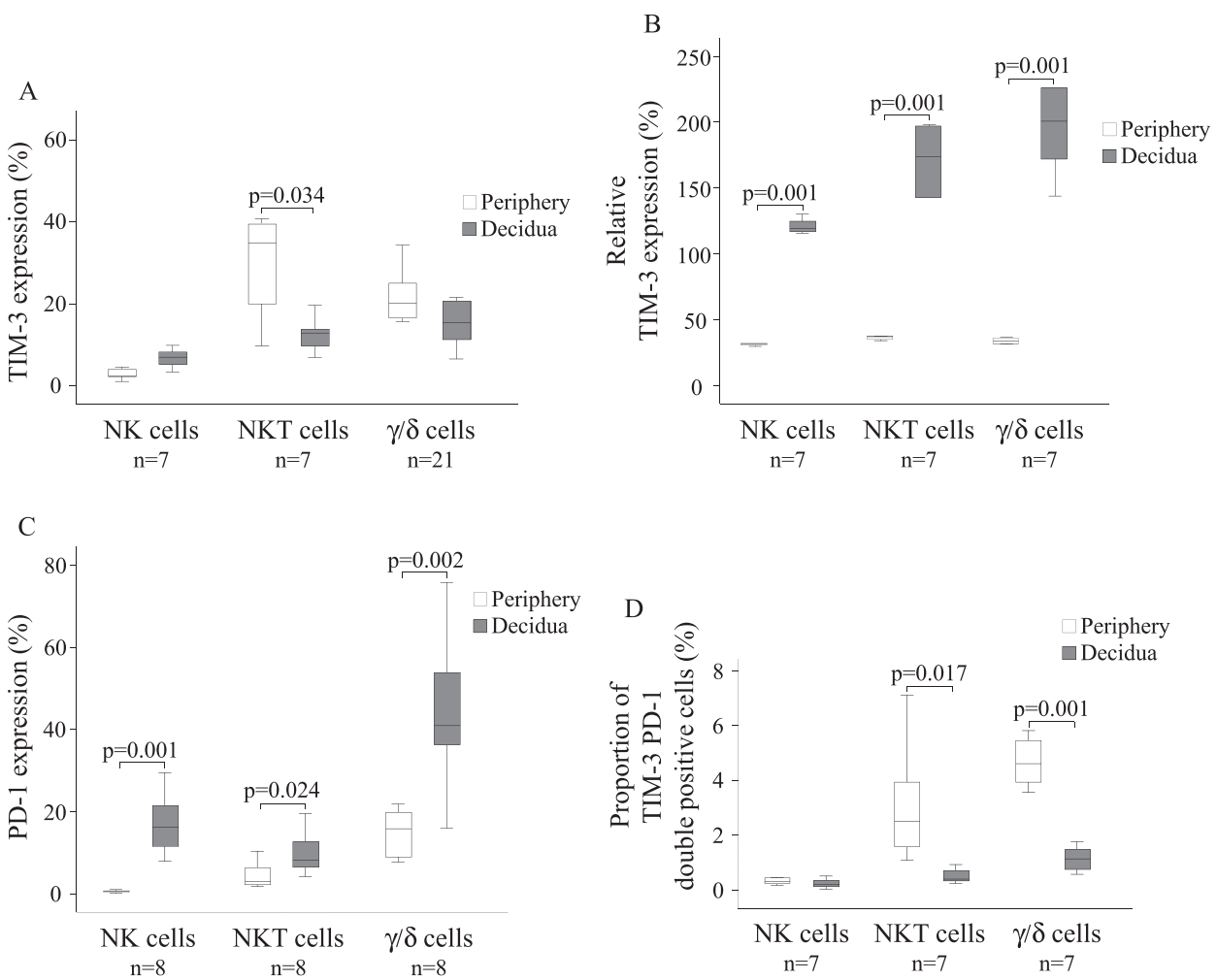

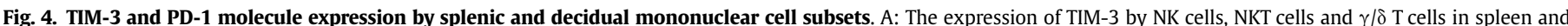

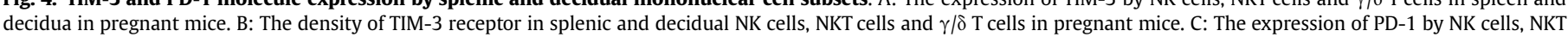

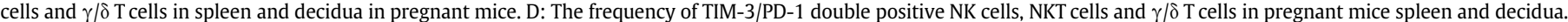

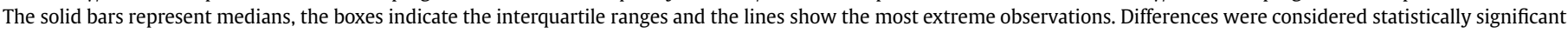
for P-values $\leq 0.05$ 

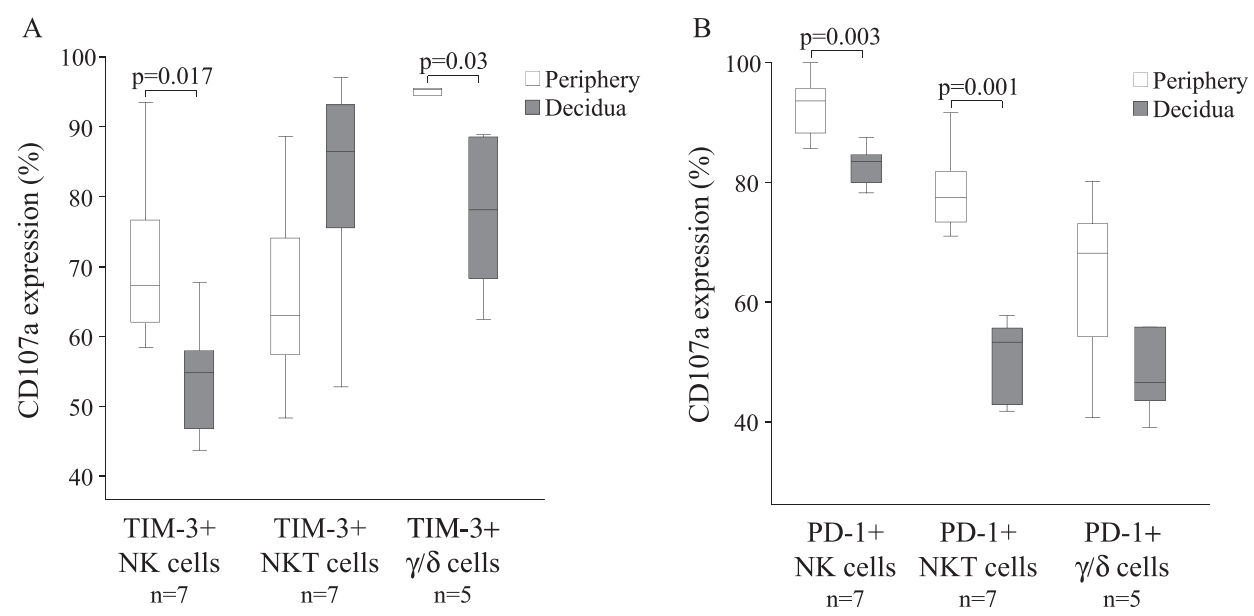

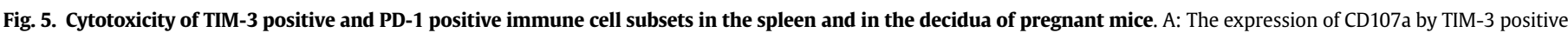

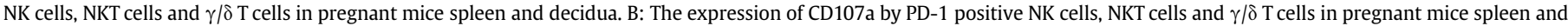

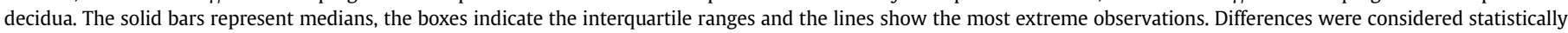
significant for P-values $\leq 0.05$.

\section{Discussion}

Immunoregulation typically manifests itself in the activation of negative pathways leading to the exhaustion of specific immune responses and cellular interactions. Co-inhibitory cell surface receptors (such as PD-1 and TIM-3) represent one of the primary tools for this purpose. PD-1 and TIM-3 have demonstrated their presence on a variety of immune cells emphasizing general involvement of these molecules in immunosuppression such as fetomaternal tolerance. Notably, the expression of their ligands is not restricted to a certain cell lineage but has been described in different tissues underlines their importance in regulating local immune responses in vivo.

Decidual expression of the ligands of PD-1 (PDL1 and PDL2) has been earlier described [28] but we report for the first time on Gal-9 expression in mouse placenta. Gal-9 was found to be present in the spongiotrophoblast layer of the haemochorial placenta, which separates the labyrinth layer from the decidua (Fig. 6). Labyrinth cells have been shown to be MHC class I and II negative, however, spongiotrophoblast expresses polymorphic paternally derived MHC class I molecules representing the fetal compartment of mouse placenta which is in direct contact with maternal decidua

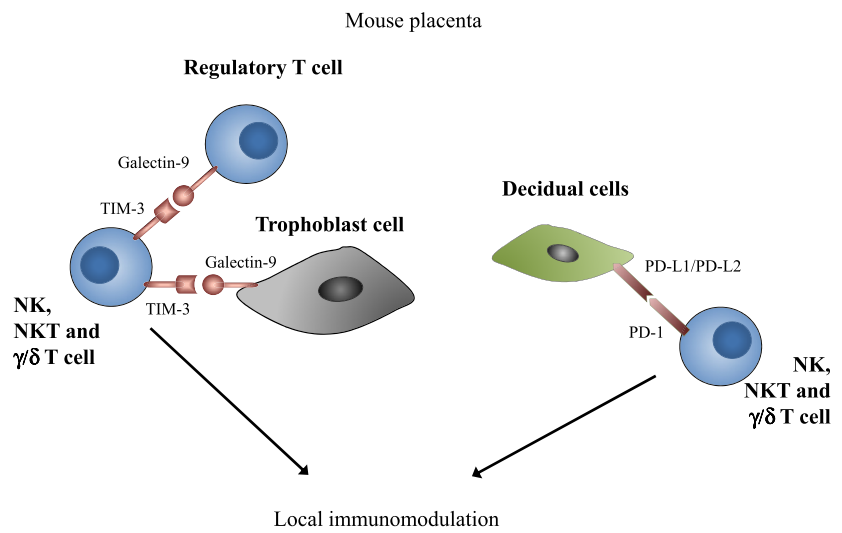

Fig. 6. Feto-maternal crosstalk via co-inhibitory molecules and their ligands. The figure represents one possible pathway of decidual NK, $\gamma / \delta \mathrm{T}$ and NKT cells involved in local immunomodulation in late mouse pregnancy. TIM-3 and PD-1 molecules are expressed by decidual NK, NKT and $\gamma / \delta$ T cells, while PDL1/PDL2 is expressed by the decidua and Galectin-9 is expressed by Tregs and by the trophoblast. enabling immune recognition of the embryo [29-31]. The other possibility of the local presence of Gal-9 is its occurrence on the maternal side. Gal-9 cell surface expression is one of the immunosuppressive tools of regulatory T cells [32]. Within the scope of our research, we discovered significantly enhanced Gal-9 expression by decidual regulatory $\mathrm{T}$ cells compared to the periphery. Moreover, there is a secreted form of Gal-9 produced by Gal-9 positive Th cells [33]. Although there is a decrease in the ratio of CD4 helper T cells in the decidua, the Gal-9 secreting subpopulation is still represented locally without any changes. According to our hypothesis, these dominant presence of the ligands PDL1, PDL2 and Gal-9 at the fetomaternal interface suggest subsequent local immunomodulatory potential following maternal immunoactivation.

Among the lymphocyte subpopulations recruited in the mouse decidua we found predominantly innate immune cells (NK cells, $\gamma /$ $\delta$ T cells and NKT cells) and a reduced number of CD4 + and CD8+ T lymphocytes compared to the periphery. These data are consistent with previous studies on decidual lymphocyte enrichment in the mouse [34-36]. Therefore, we focused on the characteristics of coinhibitory receptor expressing NK cells, $\gamma / \delta$ T cells and NKT cells (Fig. 6).

During the analysis of the PD-1 expression by lymphocytes in the periphery and in the decidua, decidual lymphocytes show a notable increased PD-1 expression in all investigated subpopulations. Cytotoxicity of the lymphocytes was measured by analyzing the expression of the degranulation marker CD107a on the cell surface. Lytic activity of PD-1 positive NK, NKT and $\gamma / \delta \mathrm{T}$ cells was decreased in the decidua, suggesting the involvement of these lymphocytes in fetomaternal tolerance as a potential result of the PD- 1 receptor mediated pathway.

While TIM- 3 expression of NK cells and $\gamma / \delta$ T cells is similar both in the periphery and in the decidua, the relative TIM-3 expression is increased locally (higher receptor density on single cell level) indicating decidual TIM-3 expressing cells are more mature and fully functional [15]. However, cytotoxic capacity of decidual TIM-3 expressing NK cells and $\gamma / \delta$ T cells is reduced when compared to the periphery which might be due to their upregulated relative TIM-3 expression on one hand and a much stronger local presence of its ligand Gal-9 on the other hand. Their binding could lead to the subsequent inhibition of effector functions observed here as a reduced cytotoxicity.

Investigating decidual NKT cells, this subpopulation showed a 
reduced TIM-3 expression with increased relative receptor expression when compared to the periphery. Cytotoxicity of these cell population increased locally, although not significantly. According to these data, there is a smaller TIM-3+ NKT cell subset in the decidua with stronger lytic capacity. Subsequently, on decidual NKT cells TIM-3 appears to promote cellular cytotoxicity than to suppress NKT cell activity hereby contributing to the mild inflammation required for successful pregnancy.

In analyzing the co-expression of PD- 1 and TIM-3 by lymphocytes we found a very small percentage of cells involved simultaneously in both inhibitory pathways. Moreover, the percentage of double positive NKT cells and $\gamma / \delta$ T cells further decreases in the decidua. Accordingly, these findings emphasize lymphocytes could be either PD-1 or TIM-3 committed, indicating separate action of these two co-inhibitory receptors at a cellular level leading to additive inhibitory effects considering whole lymphocytes populations.

Maternal immune responses triggered by the recognition of different fetal antigens mainly compose of tolerogenic mechanisms but there is also a small inflammatory component both for fetal (successful placentation and vascular remodeling) and for maternal advantages (immune protection against pathogens). These actions are mostly carried out by innate immune cells enriched in the decidua. We investigated the potential role of coinhibitory molecules PD-1 and TIM-3 in maternal immune responses, since it has been shown their ligands are present at the fetomaternal interface (Fig. 6). Our research concludes PD-1 and TIM-3 expressing decidual lymphocytes were found to be more dominant than in the periphery. While PD-1 positive lymphocytes show reduced cytotoxicity, lytic activity of TIM-3 expressing cells varies with cell type suggesting opposite roles of TIM-3 on different lymphocyte subsets.

Our results were obtained from mice at gestation day 14.5 which is rather late in mouse pregnancy so even more significant Gal-9/ TIM-3, PDL1-PDL2/PD-1 related changes could be observed earlier in pregnancy, which needs further investigations.

Comprehensively speaking, our data indicates a very complex, tissue and cell type specific immunoregulatory mechanism by the investigated co-inhibitory receptors at the fetomaternal interface proposing further investigations of their exact role in maternal immune responses.

\section{Conflict of interest}

The authors have no conflict of interest.

\section{Acknowledgments}

This work was supported by grants from Hungarian National Research Fund (OTKA K104960 and PD112465) and the Janos Bolyai Research Scholarship of the Hungarian Academy of Sciences to L. Szereday.

\section{References}

[1] L. Monney, C.A. Sabatos, J.L. Gaglia, A. Ryu, H. Waldner, T. Chernova, et al., Th1specific cell surface protein Tim-3 regulates macrophage activation and severity of an autoimmune disease [Internet] [cited 2013 Mar 12], Nature 415 (6871) (2002 Jan 31) 536-541. Available from: http://www.ncbi.nlm.nih.gov/ pubmed/11823861.

[2] A.C. Anderson, D.E. Anderson, L. Bregoli, W.D. Hastings, N. Kassam, C. Lei, et al., Promotion of tissue inflammation by the immune receptor Tim-3 expressed on innate immune cells [Internet] [cited 2013 Mar 12], Science 318 (5853) (2007 Nov 16) 1141-1143. Available from: http://www.ncbi.nlm.nih.gov/ pubmed/18006747.

[3] S. Nakae, M. Iikura, H. Suto, H. Akiba, D.T. Umetsu, R.H. Dekruyff, et al., TIM-1 and TIM-3 enhancement of Th2 cytokine production by mast cells [Internet] [cited 2014 Feb 4], Blood 110 (7) (2007 Oct 1) 2565-2568. Available from: http://www.pubmedcentral.nih.gov/articlerender.fcgi?artid=1988955\&tool= pmcentrez\&rendertype $=$ abstract.

[4] J. Lee, E.W. Su, C. Zhu, S. Hainline, J. Phuah, J.A. Moroco, et al., Phosphotyrosine-dependent coupling of Tim-3 to T-cell receptor signaling pathways [Internet] [cited 2013 Mar 6], Mol. Cell Biol. 31 (19) (2011 Oct) 3963-3974. Available from: http://www.pubmedcentral.nih.gov/articlerender.fcgi? artid $=3187355 \&$ tool $=$ pmcentrez $\&$ rendertype $=$ abstract.

[5] L. Chabtini, B. Mfarrej, M. Mounayar, B. Zhu, I. Batal, P.J. Dakle, et al., TIM-3 regulates innate immune cells to induce fetomaternal tolerance, J. Immunol. 190 (1) (2013 Jan) 88-96.

[6] N.G. Than, R. Romero, C.J. Kim, M.R. McGowen, Z. Papp, D.E. Wildman, Galectins: guardians of eutherian pregnancy at the maternal-fetal interface [Internet] [cited 2015 Feb 18], Trends Endocrinol. Metab. 23 (1) (2012 Jan) 23-31. Available from: http://www.pubmedcentral.nih.gov/articlerender. fcgi? artid=3640805\&tool $=$ pmcentrez\&rendertype $=$ abstract.

[7] E.L. Naka, V.C. Ponciano, M.A. Cenedeze, A. Pacheco-Silva, N.O.S. Câmara, Detection of the Tim-3 ligand, galectin-9, inside the allograft during a rejection episode [Internet] [cited $2013 \mathrm{Mar}$ 12], Int. Immunopharmacol. 9 (6) (2009 Jun) 658-662. Available from: http://www.ncbi.nlm.nih.gov/pubmed/ 19100864.

[8] M. Moritoki, T. Kadowaki, T. Niki, D. Nakano, G. Soma, H. Mori, et al., Galectin9 ameliorates clinical severity of MRL/lpr lupus-prone mice by inducing plasma cell apoptosis independently of Tim-3 [Internet] [cited 2014 Feb 4], PLoS One 8 (4) (2013 Jan) e60807. Available from: http://www. pubmedcentral.nih.gov/articlerender.fcgi?artid=3621869\&tool=pmcentrez\& rendertype $=$ abstract.

[9] C.A. Sabatos, S. Chakravarti, E. Cha, A. Schubart, A. Sánchez-Fueyo, X.X. Zheng et al., Interaction of Tim-3 and Tim-3 ligand regulates T helper type 1 responses and induction of peripheral tolerance [Internet] [cited 2013 Mar 12], Nat. Immunol. 4 (11) (2003 Nov) 1102-1110. Available from: http://www. ncbi.nlm.nih.gov/pubmed/14556006.

[10] C. Zhu, A.C. Anderson, A. Schubart, H. Xiong, J. Imitola, S.J. Khoury, et al., The Tim-3 ligand galectin-9 negatively regulates $\mathrm{T}$ helper type 1 immunity [Internet] [cited 2014 Feb 4], Nat. Immunol. 6 (12) (2005 Dec) 1245-1252. Available from: http://www.ncbi.nlm.nih.gov/pubmed/16286920.

[11] M. Seki, S. Oomizu, K.-M. Sakata, A. Sakata, T. Arikawa, K. Watanabe, et al., Galectin-9 suppresses the generation of Th17, promotes the induction of regulatory $\mathrm{T}$ cells, and regulates experimental autoimmune arthritis [Internet] [cited 2013 Mar 12], Clin. Immunol. 127 (1) (2008 Apr) 78-88. Available from: http://www.ncbi.nlm.nih.gov/pubmed/18282810.

[12] Z.-H. Tang, S. Liang, J. Potter, X. Jiang, H.-Q. Mao, Z. Li, Tim-3/galectin-9 regulate the homeostasis of hepatic NKT cells in a murine model of nonalcoholic fatty liver disease [Internet] [cited 2014 Feb 4], J. Immunol. 190 (4) (2013 Feb 15) 1788-1796. Available from: http://www.pubmedcentral. nih.gov/articlerender.fcgi ?artid=3563933\&tool=pmcentrez\&rendertype $=$ abstract.

[13] W.D. Hastings, D.E. Anderson, N. Kassam, K. Koguchi, E.A. Greenfield, S.C. Kent, et al., TIM-3 is expressed on activated human CD4+ T cells and regulates Th1 and Th17 cytokines [Internet] [cited 2015 Jan 30], Eur. J. Immunol. 39 (9) (2009 Sep) 2492-2501. Available from: http://www.pubmedcentral.nih.gov/ articlerender.fcgi? artid $=2759376 \&$ tool $=$ pmcentrez\&rendertype $=$ abstract.

[14] Y. Ju, N. Hou, J. Meng, X. Wang, X. Zhang, D. Zhao, et al., T cell immunoglobulin- and mucin-domain-containing molecule-3 (Tim-3) mediates natural killer cell suppression in chronic hepatitis B [Internet] [cited 2015 Feb 9], J. Hepatol. 52 (3) (2010 Mar) 322-329. Available from: http://www.ncbi.nlm. nih.gov/pubmed/20133006.

[15] L.C. Ndhlovu, S. Lopez-Vergès, J.D. Barbour, R.B. Jones, A.R. Jha, B.R. Long, et al., Tim-3 marks human natural killer cell maturation and suppresses cellmediated cytotoxicity [Internet] [cited 2015 Feb 9], Blood 119 (16) (2012 Apr 19) 3734-3743. Available from: http://www.pubmedcentral.nih.gov/ articlerender.fcgi? artid=3335380\&tool=pmcentrez\&rendertype=abstract.

[16] M.K. Gleason, T.R. Lenvik, V. McCullar, M. Felices, M.S. O'Brien, S.A. Cooley, et al., Tim-3 is an inducible human natural killer cell receptor that enhances interferon gamma production in response to galectin-9 [Internet] [cited 2015 Feb 9], Blood 119 (13) (2012 Mar 29) 3064-3072. Available from: http:// www.pubmedcentral.nih.gov/articlerender.fcgi? artid=3321868\&tool=pmcen trez\&rendertype $=$ abstract.

[17] C.A.M. Finney, K. Ayi, J.D. Wasmuth, P.M. Sheth, R. Kaul, M.R. Loutfy, et al., HIV infection deregulates innate immunity to malaria despite combination antiretroviral therapy [Internet] [cited 2015 Feb 9], AIDS 27 (3) (2013 Jan 28) 325-335. Available from: http://www.ncbi.nlm.nih.gov/ pubmed/23291537.

[18] C.A.M. Finney, K. Ayi, J.D. Wasmuth, P.M. Sheth, R. Kaul, M. Loutfy, et al., HIV infection deregulates Tim-3 expression on innate cells: combination antiretroviral therapy results in partial restoration [Internet] [cited 2015 Feb 9], J. Acquir Immune Defic. Syndr. 63 (2) (2013 Jun 1) 161-167. Available from: http://www.ncbi.nlm.nih.gov/pubmed/23314411.

[19] M.E. Keir, M.J. Butte, G.J. Freeman, A.H. Sharpe, PD-1 and its ligands in tolerance and immunity [Internet] [cited 2014 May 27], Annu. Rev. Immunol. 26 (2008 Jan) 677-704. Available from: http://www.ncbi.nlm.nih.gov/pubmed/ 18173375.

[20] M.N. Wykes, J.M. Horne-Debets, C.-Y. Leow, D.S. Karunarathne, Malaria drives T cells to exhaustion [Internet] [cited 2014 Jun 10], Front. Microbiol. 5 (2014 Jan) 249. Available from: http://www.pubmedcentral.nih.gov/articlerender. fcgi artid $=4034037 \&$ tool $=$ pmcentrez\&rendertype $=$ abstract. 
[21] H. Dong, G. Zhu, K. Tamada, L. Chen, B7-H1, a third member of the B7 family, co-stimulates T-cell proliferation and interleukin-10 secretion [Internet] [cited 2014 Jun 10], Nat. Med. 5 (12) (1999 Dec) 1365-1369. Available from: http://www.ncbi.nlm.nih.gov/pubmed/10581077.

[22] G.J. Freeman, A.J. Long, Y. Iwai, K. Bourque, T. Chernova, H. Nishimura, et al., Engagement of the PD-1 immunoinhibitory receptor by a novel B7 family member leads to negative regulation of lymphocyte activation [Internet] [cited 2014 Jun 3], J. Exp. Med. 192 (7) (2000 Oct 2) 1027-1034. Available from: http://www.pubmedcentral.nih.gov/articlerender.fcgi?artid=2193311 \&tool=pmcentrez\&rendertype $=$ abstract.

[23] Y. Latchman, C.R. Wood, T. Chernova, D. Chaudhary, M. Borde, I. Chernova, et al., PD-L2 is a second ligand for PD-1 and inhibits T cell activation [Internet] [cited 2014 Jun 3], Nat. Immunol. 2 (3) (2001 Mar) 261-268. Available from: http://www.ncbi.nlm.nih.gov/pubmed/11224527.

[24] A.H. Sharpe, E.J. Wherry, R. Ahmed, G.J. Freeman, The function of programmed cell death 1 and its ligands in regulating autoimmunity and infection [Internet] [cited 2014 Jun 4], Nat. Immunol. 8 (3) (2007 Mar) 239-245. Available from: http://www.ncbi.nlm.nih.gov/pubmed/17304234.

[25] E.J. Wherry, T cell exhaustion [Internet] [cited 2015 Jan 14], Nat. Immunol. 12 (6) (2011 Jun) 492-499. Available from: http://www.ncbi.nlm.nih.gov/ pubmed/21739672.

[26] J. Leitner, A. Rieger, W.F. Pickl, G. Zlabinger, K. Grabmeier-Pfistershammer, P. Steinberger, TIM-3 does not act as a receptor for galectin-9 [Internet] [cited 2015 Feb 9], PLoS Pathog. 9 (3) (2013 Mar) e1003253. Available from: http:// www.pubmedcentral.nih.gov/articlerender.fcgi?artid $=3605152 \&$ tool $=$ pm centrez\&rendertype =abstract.

[27] M.W. Pfaffl, G.W. Horgan, L. Dempfle, Relative expression software tool (REST) for group-wise comparison and statistical analysis of relative expression results in real-time PCR [Internet] [cited 2014 Feb 4], Nucleic Acids Res. 30 (9) (2002 May 1) e36. Available from: http://www.pubmedcentral.nih.gov/ articlerender.fcgi?artid=113859\&tool=pmcentrez\&rendertype=abstract.

[28] I. Guleria, A. Khosroshahi, M.J. Ansari, A. Habicht, M. Azuma, H. Yagita, et al., A critical role for the programmed death ligand 1 in fetomaternal tolerance [Internet] [cited 2015 Feb 10], J. Exp. Med. 202 (2) (2005 Jul 18) 231-237. Available from: http://www.pubmedcentral.nih.gov/articlerender.fcgi?artid= $2213002 \&$ tool $=$ pmcentrez\&rendertype $=$ abstract.
[29] R.W. Redline, C.Y. Lu, Localization of fetal major histocompatibility complex antigens and maternal leukocytes in murine placenta. Implications for maternal-fetal immunological relationship [Internet] [cited 2015 Feb 10], Lab. Invest 61 (1) (1989 Jul) 27-36. Available from: http://www.ncbi.nlm.nih.gov/ pubmed/2473277.

[30] A. Moffett, C. Loke, Immunology of placentation in eutherian mammals [Internet] [cited 2015 Feb 10], Nat. Rev. Immunol. 6 (8) (2006 Aug) 584-594 Available from: http://www.ncbi.nlm.nih.gov/pubmed/16868549.

[31] F.A. Zuckermann, J.R. Head, Expression of MHC antigens on murine trophoblast and their modulation by interferon [Internet] [cited 2015 Feb 10] J. Immunol. 137 (3) (1986 Aug 1) 846-853. Available from: http://www.ncbi. nlm.nih.gov/pubmed/2424987.

[32] F. Wang, L. Wan, C. Zhang, X. Zheng J. Li, Z.K. Chen, Tim-3-Galectin-9 pathway involves the suppression induced by CD4+CD25+ regulatory T cells [Internet] [cited 2015 Jan 29], Immunobiology 214 (5) (2009 Jan) 342-349. Available from: http://www.ncbi.nlm.nih.gov/pubmed/19362679.

[33] S. Oomizu, T. Arikawa, T. Niki, T. Kadowaki, M. Ueno, N. Nishi, et al., Cel surface galectin-9 expressing Th cells regulate Th17 and Foxp3+ Treg development by galectin-9 secretion [Internet] [cited 2014 Dec 9], PLoS One 7 (11) (2012 Jan) e48574. Available from: http://www.pubmedcentral. nih.gov/articlerender.fcgi? artid=3492452\&tool=pmcentrez\&rendertype=abstract.

[34] L. Mincheva-Nilsson, Pregnancy and gamma/delta T cells: taking on the hard questions [Internet] [cited 2015 Feb 10], Reprod. Biol. Endocrinol. 1 (2003 Dec 2) 120. Available from: http://www.pubmedcentral.nih.gov/articlerender. fcgi?artid=305336\&tool=pmcentrez\&rendertype $=$ abstract.

[35] S.M. Blois, B.F. Klapp, G. Barrientos, Decidualization and angiogenesis in early pregnancy: unravelling the functions of DC and NK cells [Internet] [cited 2015 Feb 10], J. Reprod. Immunol. 88 (2) (2011 Mar) 86-92. Available from: http:// www.ncbi.nlm.nih.gov/pubmed/21227511.

[36] K. Ito, M. Karasawa, T. Kawano, T. Akasaka, H. Koseki, Y. Akutsu, et al., Involvement of decidual Valpha14 NKT cells in abortion [Internet] [cited 2015 Feb 10], Proc. Natl. Acad. Sci. U. S. A. 97 (2) (2000 Jan 18) 740-744. Available from: http://www.pubmedcentral.nih.gov/articlerender.fcgi?artid=15400\& tool=pmcentrez\&rendertype $=$ abstract. 